\title{
Avaliação da atividade antifúngica do óleo essencial de Pogostemon cablin (Blanco) Benth. (Lamiaceae) contra cepas de Candida glabrata
}

Evaluation of the antifungal activity of the essential oil of Pogostemon cablin (Blanco) Benth. (Lamiaceae) against Candida glabrata

\author{
E. S. Pimenta ${ }^{1}$; R. M. C. da Cruz ${ }^{1}$; H. Diniz Neto ${ }^{2 *}$; D. F. Silva ${ }^{2}$; H. M. B. F. de \\ Oliveira $^{3}$; D. F. Buccini ${ }^{4}$; E. O. Lima ${ }^{2}$; A. A. de Oliveira Filho ${ }^{5}$ \\ ${ }^{1}$ Secretaria Municipal de Saúde de Paulo Afonso, Centro, 48603-040, Paulo Afonso - BA, Brasil. \\ ${ }^{2}$ Universidade Federal da Paraíba, Departamento de Ciências Farmacêuticas, Centro de Ciências da Saúde, Castelo \\ Branco III, 58059-900, João Pessoa- PB, Brasil. \\ ${ }^{3}$ Maternidade e Hospital Universitário Ana Bezerra, Universidade Federal do Rio Grande do Norte, Praça Manoel \\ Villaça, 59200-000, Santa Cruz - RN, Brasil. \\ ${ }^{4}$ Pós-Graduação em Farmacologia, Universidade Católica Dom Bosco, Jardim Seminário, 79117-900, Campo Grande \\ - MS, Brasil. \\ ${ }^{5}$ Centro de Saúde e Tecnologia Rural, Universidade Federal de Campina Grande, Jatobá, 58700-970, Patos - PB, \\ Brasil. \\ *hermes.dn@hotmail.com
}

(Recebido em 28 agosto de 2018; aceito em 30 de junho de 2019)

\begin{abstract}
A candidíase invasiva é uma ameaça crescente entre pacientes imunocomprometidos, sendo uma causa significativa de morbidade e mortalidade. Epidemiologicamente, as espécies não-albicans estão adquirindo relevância por se tornarem os agentes causadores cada vez mais frequentes de candidíase. O uso indiscriminado de medicamentos para combater as diversas variedades de candidíase tem trazido um aumento expressivo no número de leveduras resistentes aos antifúngicos tradicionais. Desta forma, produtos de origem vegetal podem ser utilizados como alternativa para os antifúngicos sintéticos. Com base nestas informações, esta pesquisa objetivou avaliar a possível atividade antifúngica do óleo essencial de Pogostemon cablin (Blanco) Benth. (Lamiaceae) contra cepas de Candida glabrata através da técnica de microdiluição para a determinação da Concentração Inibitória Mínima (CIM) e Concentração Fungicida Mínima (CFM). Após o experimento, observou-se que o produto inibiu a cepa LM-302 de C. glabrata com uma CIM de $256 \mu \mathrm{g} / \mathrm{mL}$. $\mathrm{O}$ mesmo valor foi obtido para a CFM contra esta mesma cepa. Portanto, pode-se concluir que o óleo essencial de $P$. cablin testado apresenta um efeito antifúngico contra as leveduras de $C$. glabrata.

Palavras-chave: Candida não-albicans, fitoterapia, Pogostemon cablin.
\end{abstract}

Invasive candidiasis is a growing threat among critically ill patients and is a significant cause of morbidity and mortality. Epidemiologically, non-albicans species are becoming relevant because they are becoming the most frequent causative agents of candidiasis. The indiscriminate use of drugs to combat the various strains of candidiasis has brought an expressive increase in the number of drug resistant yeasts. In this way, products of plant origin can be used as a substitute for synthetic antifungals agents. Based on this information, this research aimed to evaluate the possible antifungal activity of Pogostemon cablin (Blanco) Benth. (Lamiaceae) essential oil against Candida glabrata strains by microdilution technique for the determination of Minimum Inhibitory Concentration (MIC) and Minimum Fungicidal Concentration (MFC). After the experiment, the product was found to inhibit the C. glabrata strain LM-302 with a MIC of $256 \mu \mathrm{g} / \mathrm{ml}$. The same value was obtained for MFC against this same strain. Therefore, it can be concluded that the essential oil of $P$. cablin tested has an antifungal effect against the yeasts of $C$. glabrata.

Keywords: Candida non-albicans, phytotherapy, Pogostemon cablin.

\section{INTRODUÇÃO}

As leveduras do gênero Candida são responsáveis por $80 \%$ das infecções fúngicas sistêmicas, representando $17 \%$ dos casos de infecções em unidades de terapia intensiva. Além de possuir 
elevada taxa de mortalidade, as candidíases sistêmicas aumentam o tempo de permanência em hospitais, resultando em aumento de gastos relacionados ao tratamento [1,2].

Mesmo que Candida albicans permaneça como a espécie majoritariamente associada à candidíase, espécies de Candida não-albicans são responsáveis por uma quantidade crescente de infecções, tais como: infecções torácicas, empiema e infecção traqueobrônquica com disseminação para os pulmões em pacientes imunocomprometidos [3].

Dentre os grandes obstáculos para o tratamento das micoses humanas, pode-se destacar: a crescente resistência aos fármacos antifúngicos, opções terapêuticas limitadas, toxicidade, interações medicamentosas e biodisponibilidade diminuta das drogas antifúngicas presentes no mercado atual [4-7].

Diante desta premissa surge grande necessidade de desenvolvimento de novos fármacos com maior eficácia e segurança, e nesta lógica, voltam-se os olhares para os fitoterápicos como um meio alternativo de tratamento. As vantagens dos fitoterápicos frente aos medicamentos sintéticos está na grande variedade molecular, visto que inúmeros compostos derivados de plantas medicinais têm sido utilizados como protótipos para a semi-síntese de novos fármacos antimicrobianos, menos tóxicos e mais eficientes, o que proporciona um campo vasto para descoberta de novos fármacos para o tratamento de casos de candidíase $[8,9]$.

A Pogostemon cablin (Blanco) Benth., popularmente conhecida como patchouli, pertence à família Lamiaceae, sendo esta uma planta do tipo arbustiva, perene e adaptada ao clima quente e úmido. A P. cablin é bastante cultivada na Indonésia, China, Singapura, Índia e América do Sul devido a sua capacidade de armazenar óleos essenciais em suas folhas, apresentando grande valor comercial [10]. Farmacologicamente, a $P$. cablin já demonstrou possuir ampla atividade farmacológica, entre elas: anti-emética [11], antitripanossômica [12] e inseticida [13].

Sendo assim, diante da necessidade urgente na descoberta de novas alternativas terapêuticas para combater as leveduras do gênero Candida, que geram grandes prejuízos a saúde da população, o presente trabalho busca avaliar a possível atividade antifúngica do óleo essencial de $P$. cablin contra cepas de C. glabrata.

\section{MATERIAL E MÉTODOS}

O óleo essencial de $P$. cablin foi adquirido da empresa Herbia ${ }^{\circledR}$. Como antifúngico padrão, foi utilizado anfotericina B (Pharma Nostra, Rio de Janeiro). As emulsões das substâncias utilizadas nos ensaios foram preparadas no momento de execução dos ensaios. As emulsões do óleo essencial e da anfotericina B foram preparadas solubilizando as substâncias em tween 80 a $2 \%$ e dimetilsulfóxido (DMSO) a 5\%, completando o volume final com água destilada esterilizada para obter uma emulsão na concentração de $1024 \mu \mathrm{g} / \mathrm{mL}$.

Foram utilizadas quatro cepas de Candida glabrata (LM-L6, LM-17, LM-116 e LM 302), previamente isoladas e identificadas, cedidas pelo Laboratório de Micologia do Departamento de Ciências Farmacêuticas, Centro de Ciências da Saúde, Universidade Federal da Paraíba. Todas as cepas foram mantidas em ágar Sabouraud dextrose (ASD) a uma temperatura de $4^{\circ} \mathrm{C}$, sendo utilizados para os ensaios repiques de 24 horas em ASD incubados a $35^{\circ} \mathrm{C}$. Para preparação do inóculo, colônias obtidas de culturas das cepas de Candida spp. mantidas em ASD, foram suspensas em solução de $\mathrm{NaCl} 0,85 \%$ estéril e ajustadas de acordo com o padrão 0,5 de McFarland $\left(10^{6}\right.$ $\mathrm{UFC} / \mathrm{mL})[14]$.

A concentração inibitória mínima do óleo essencial de $P$. cablin foi determinada pela técnica de microdiluição em caldo $[14,15]$. Foram utilizadas placas de poliestireno de 96 poços estéreis com fundo em "U" e com tampa. Em cada poço da placa, foram adicionados $100 \mu \mathrm{L}$ do meio RPMI 1640. Em seguida, $100 \mu \mathrm{L}$ da emulsão do óleo essencial de $P$. cablin foram dispensados nas cavidades da primeira linha da placa. Por meio de uma diluição seriada em razão de dois, foram obtidas as concentrações de 1024 a $4 \mu \mathrm{g} / \mathrm{mL}$. Depois, foram adicionados $10 \mu \mathrm{L}$ do inóculo das cepas fúngicas nas cavidades. Por fim, as placas foram assepticamente fechadas e incubadas a incubadas à $37^{\circ} \mathrm{C}$ por $24-48$ horas. A CIM foi definida como a menor concentração do produto capaz de produzir inibição visual do crescimento microbiano nos poços em comparação com os controles através da observação a olho nu da ausência de turvação do meio no fundo do poço. 
Paralelamente, foram realizados os controles com antifúngico padrão (anfotericina B 100 $\mu \mathrm{g} / \mathrm{mL}$ ), de esterilidade do meio (poços da microplaca contendo apenas o meio líquido RPMI 1640) e de viabilidade dos micro-organismos (poços da microplaca contendo apenas o caldo e o inóculo das leveduras). Para verificar a ausência de interferência nos resultados pelos solventes utilizados na preparação da emulsão (DMSO e Tween 80), foi feito um controle no qual foram colocados nas cavidades da microplaca o meio RPMI 1640, DMSO a 5\%, Tween 80 a $2 \%$ e o inóculo fúngico. Os ensaios foram realizados em duplicata e os resultados expressos como a média aritmética das CIM obtidas.

A concentração fungicida mínima (CFM) do óleo essencial de $P$. cablin contra as cepas de $C$. glabrata também foi determinada. Após a leitura da CIM em 48 horas, alíquotas de $20 \mu \mathrm{L}$ foram retiradas de cada poço da placa de microdiluição que não apresentou crescimento fúngico, e transferidas para poços de uma nova placa contendo apenas $100 \mu \mathrm{L}$ do meio RPMI 1640. As placas inoculadas foram assepticamente fechadas e incubadas a $35^{\circ} \mathrm{C}$, e as CFMs foram registradas após 48h. A CFM foi definida como a menor concentração do óleo essencial onde não houve crescimento de leveduras, através da observação a olho nu da ausência de turvação do meio no fundo do poço, após o período de incubação [16].

\section{RESULTADOS E DISCUSSÃO}

Os resultados referentes à ação antifúngica do óleo essencial de $P$. cablin contra cepas de $C$. glabrata encontram-se na Tabela 1.

Tabela 1: Concentração inibitória mínima (CIM) e concentração fungicida mínima (CFM) do óleo essencial de Pogostemon cablin e Anfotericina B (Anf) sobre cepas das espécies de Candida glabrata

(C.g.).

\begin{tabular}{|c|c|c|c|c|c|}
\hline \multirow[t]{2}{*}{ Leveduras } & \multicolumn{2}{|c|}{$\begin{array}{c}\text { Óleo essencial de Pogostemon } \\
\text { cablin }(\mu \mathrm{g} / \mathrm{mL})\end{array}$} & \multirow{2}{*}{$\begin{array}{c}\text { Anf } \\
(100 \mu \mathrm{g} / \mathrm{mL})\end{array}$} & \multirow{2}{*}{$\begin{array}{l}\text { Controle de } \\
\text { esterilidade }\end{array}$} & \multirow{2}{*}{$\begin{array}{l}\text { Controle de } \\
\text { crescimento }^{\mathrm{a}}\end{array}$} \\
\hline & CIM & CFM & & & \\
\hline C. g. LM-L6 & ND & ND & - & - & + \\
\hline C. g. LM-17 & ND & ND & - & - & + \\
\hline C. g. LM-116 & ND & ND & - & - & + \\
\hline C. g. LM-302 & 256 & 256 & - & - & + \\
\hline
\end{tabular}

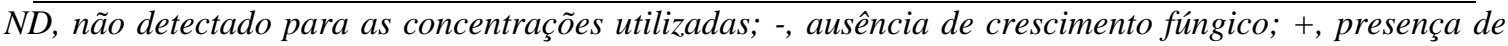
crescimento fúngico; ${ }^{a}$ crescimento fúngico em RPMI-1640, DMSO (5\%) e Tween 80 (2\%), sem agente antifúngico.

O óleo essencial de P. cablin inibiu apenas uma das cepas testadas (LM-302), para as demais cepas observa-se que a maior concentração testada, $1024 \mu \mathrm{g} / \mathrm{mL}$ não foi capaz de inibir o crescimento fúngico. Os valores obtidos para a CFM do óleo essencial frente as cepas clínicas de C. glabrata foram semelhantes aos valores da CIM, inibindo apenas a cepa (LM-302) na concentração de $256 \mu \mathrm{g} / \mathrm{mL}$. A ação do produto restrita a apenas uma das cepas testadas pode ser resultado de resistência adquirida por parte das outras cepas, visto que, por serem de origem clínica, estas podem ter sido expostas a um tratamento antifúngico ineficaz que promoveu $\mathrm{o}$ desenvolvimento de resistência [17].

Liu et al. (2012) [18], ao estudar mais de 100 ensaios clínicos para tratar vaginite micótica causada por Candida sp., observou que os extratos etanólico, hexânico e acetônico de P. cablin apresentaram efeito antifúngico para as cepas testadas de C. albicans, comparando-se clinicamente ao antifúngico miconazol.

Yi et al. (2013) [19], ao avaliar a atividade antifúngica da pogostona, um dos metabólitos segundários de $P$. cablin, e uma série de análogos deste metabólito, observou forte atividade antifúngica da pogostona e de um dos análogos sobre todas as cepas de $C$. albicans utilizadas no teste.

Anteriormente, Salari et al. (2016) [20] avaliou as propriedades antifúngicas dos extratos metanólicos de Salvia rhytidea Benth (Lamiaceae) contra cepas de $C$. albicans, $C$. parapsilosis, $C$. glabrata, C. tropicalis, C. lusitaniae, C. krusei e C. guilliermondii, utilizando um método de 
microdiluição em caldo. Os resultados revelaram que os isolados de $C$. glabrata foram susceptíveis, apesar de que os extratos foram mais eficazes contra os isolados de $C$. albicans, $C$. tropicalis e $C$. krusei.

O potencial antimicrobiano de uma determinada substância é considerado forte quando a CIM atinge valores inferiores a $0,5 \mathrm{mg} / \mathrm{mL}$, moderado entre $0,6-1,5 \mathrm{mg} / \mathrm{mL}$ e fraco quando ultrapassam $1,5 \mathrm{mg} / \mathrm{mL}$ [21,22]. O óleo essencial de $P$. cablin pode ser considerado forte para a cepa LM-302 de $C$. glabrata. Entretanto, para as outras cepas, o óleo essencial não pode ser classificado, visto que não apresentou atividade antifúngica dentro das concentrações utilizadas no teste.

Lund et al. (2012) [23] testou a capacidade antifúngica do extrato hidroalcoólico de Mentha arvensis, pertencente a família Lamiaceae, contra cepas de $C$. albicans realizando três ensaios com métodos diferentes (métodos de difusão em ágar, macro e microdiluição em caldo). $\mathrm{O}$ autor em questão concluiu que o extrato de Mentha arvensis foi capaz de inibir o crescimento das cepas fúngicas de C. albicans.

Estudos de Lima et al. (2006) [24] revelaram susceptibilidade de cepas de C. krusei e $C$. tropicalis para o óleo essencial de outra espécie vegetal pertencente à família Lamiaceae, a Peumus boldus. Neste mesmo estudo também puderam observar que apenas uma cepa de $C$. krusei foi inibida sob ação do óleo essencial de Rosmarinus officinalis, outra planta da família Lamiaceae.

\section{CONCLUSÃO}

Com a realização deste estudo pode-se considerar que a bioatividade do óleo essencial de $P$. cablin evidenciada contra uma levedura sensível de $C$. glabrata permite vislumbrar seu potencial bioativo contra demais fungos patogênicos quer sejam leveduras ou fungos filamentosos. No entanto, mais estudos microbiológicos e farmacológicos in vitro e in vivo precisam ser feitos para a interpretação do mecanismo de ação deste produto natural de potencial antifúngico.

\section{REFERÊNCIAS BIBLIOGRÁFICAS}

1. Canela HMS, Cardoso B, Vitali LH, Coelho HC, Martinez R, Ferreira MES. Prevalence, virulence factors and antifungal susceptibility of Candida spp. isolated from bloodstream infections in a tertiary care hospital in Brazil. Mycoses. 2017;61(1):11-21. doi:10.1111/myc.12695.

2. Freire JCP, Nóbrega MTC, Freire SCP, Dias-Ribeiro E. Candidíase oral em usuários de próteses dentárias removíveis: fatores associados. Arch Health Investig. 2017;6(4). doi:10.21270/archi.v6i4.1923.

3. Jain A, Rawat SK, Rai A. Rising Incidence of Non-albicans Candida and Changing Susceptibility Pattern of bloodstream Candida Isolates in Nenates. J Clin Diagn res. 2017;11(11):1-4

4. Barberán J, Mensa J, Fariñas C, Llinares P, Serrano R, Menéndez R, et al. Recommendations of antifungal treatment in patients with low grade immunosuppression. Rev Espanola Quimioter Publicacion Of Soc Espanola Quimioter. 2008;21(2):127-42.

5. Cavaleiro C, Pinto E, Gonçalves M, Salgueiro L. Antifungal activity of Juniperus essential oils against dermatophyte, Aspergillus and Candida strains. J Appl Microbiol. 2006;100(6):1333-8. doi: 10.1111/j.1365-2672.2006.02862.x.

6. Silva FM, De Paula JE, Espindola LS. Evaluation of the antifungal potential of Brazilian Cerrado medicinal plants. Mycoses. 2009;52(6):511-7. doi: 10.1111/j.1439-0507.2008.01647.x.

7. Bhattacharya A, Kaur H. Phytochemicals as Antimicrobial Agents Against Opportunistic Candidiasis: A Review. 2015;7(3):440-5.

8. Andrade MA, das Graças Cardoso M, Batista LR, Mallet ACT, Machado SMF. Óleos essenciais de Cymbopogon nardus, Cinnamomum zeylanicum e Zingiber officinale: composição, atividades antioxidante e antibacteriana. Rev Ciênc Agronômica. 2012;43(2):399-408.

9. Barbosa-Filho JM, Nascimento Júnior FA do, Tomaz AC de A, Athayde-Filho PF de, Silva MS da, Cunha EV, et al. Natural products with antileprotic activity. Rev Bras Farmacogn. 2007;17(1):141-8. doi: 10.1590/S0102-695X2007000100022.

10. Blank AF, Sant'ana TCP, Santos PS, Arrigoni-Blank MF, do Nascimento Prata AP, Jesus HCR, et al. Chemical characterization of the essential oil from patchouli accessions harvested over four seasons. Ind Crops Prod. 2011;34(1):831-7. doi: 10.1016/j.indcrop.2011.01.021. 
11. Yang Y, Kinoshita K, Koyama K, Takahashi K, Tai T, Nunoura Y, Watanabe K. Anti-emetic principles of Pogostemon cablin (Blanco) Benth. Phytomedicine. 1999;6(2):89-93. doi: 10.1016/S09447113(99)80041-5.

12. Kiuchi F, Matsuo K, Ito M, Qui TK, Honda G. New sesquiterpene hydroperoxides with trypanocidal activity from Pogostemon cablin. Chem Pharm Bull. 2004;52(12):1495-1496. doi: $10.1248 / \mathrm{cpb} .52 .1495$.

13. Albuquerque EL, Lima JK, Souza FH, Silva IM, Santos AA, Araújo APA, et al. Insecticidal and repellence activity of the essential oil of Pogostemon cablin against urban ants species. Acta Trop. 2013;127(3):181-6. doi: 10.1016/j.actatropica.2013.04.011.

14. Cleeland R, Squires E. Evaluation of new antimicrobials in vitro and in experimental animal infections. Antibiot Lab Med. 1991;3:739-87.

15. Hadacek F, Greger H. Testing of antifungal natural products: methodologies, comparability of results and assay choice. Phytochem Anal. 2000;11(3):137-47. doi: 10.1002/(SICI)10991565(200005/06)11:3<137::AID-PCA514>3.0.CO;2-.

16. Ncube N, Afolayan A, Okoh A. Assessment techniques of antimicrobial properties of natural compounds of plant origin: current methods and future trends. Afr J Biotechnol. 2008;7(12). doi: 10.5897/AJB07.613.

17. Gonçalves SS, Souza ACR, Chowdhary A, Meis JF, Colombo AL. Epidemiology and molecular mechanisms of antifungal resistance in Candida and Aspergillus. Mycoses. abril de 2016;59(4):198219. doi: $10.1111 /$ myc.12469.

18. Liu Q, Luyten W, Pellens K, Wang Y, Wang W, Thevissen K, et al. Antifungal activity in plants from Chinese traditional and folk medicine. J Ethnopharmacol. 2012; 143(3):772-8. doi: 10.1016/j.jep.2012.06.019.

19. Yi Y-Y, He J-J, Su J-Q, Kong S-Z, Su J-Y, Li Y-C, et al. Synthesis and antimicrobial evaluation of pogostone and its analogues. Fitoterapia. 2013;84:135-9. doi: 10.1016/j.fitote.2012.11.005.

20. Salari S, Bakhshi T, Sharififar F, Naseri A, Almani PGN. Evaluation of antifungal activity of standardized extract of Salvia rhytidea Benth.(Lamiaceae) against various Candida isolates. J Mycol Medicale. 2016; 26(4):323-30. doi: 10.1016/j.mycmed.2016.06.003.

21. Sartoratto A, Machado ALM, Delarmelina C, Figueira GM, Duarte MCT, Rehder VLG. Composition and antimicrobial activity of essential oils from aromatic plants used in Brazil. Braz J Microbiol. 2004;35(4):275-80. doi: 10.1590/S1517-83822004000300001.

22. Aligiannis N, Kalpoutzakis E, Mitaku S, Chinou IB. Composition and antimicrobial activity of the essential oils of two Origanum species. J Agric Food Chem. 2001;49(9):4168-4170. doi: $10.1021 / \mathrm{jf001494m}$.

23. Lund RG, Serpa R, da Silva Nascente P, Ribeiro GA, Freitag RA, Del Pino FAB. In vitro study on the antimicrobial effect of hydroalcoholic extracts from Mentha arvensis L.(Lamiaceae) against oral pathogens. Acta Sci Biol Sci. 2012; 34(4):437-42. doi: 10.4025/ actascibiolsci.v34i4.8959.

24. de Oliveira Lima I, Oliveira R, de Oliveira Lima E, Farias NMP, de Souza EL. Atividade antifúngica de óleos essenciais sobre espécies de Candida. Braz J Pharmacogn. 2006; 16(2):197-201. doi: 10.1590/S0102-695X2006000200011. 